\title{
Feasibility of STAP for passive GSM-based radar
}

\author{
Xavier Neyt*, Jacques Raout ${ }^{\dagger}$, Mireille Kubica*, Virginie Kubica*, \\ Serge Roques ${ }^{\ddagger}$, Marc Acheroy*, Jacques G. Verly ${ }^{\S}$ \\ * Electrical Engineering Dept., Royal Military Academy, Belgium, xavier.neyt@elec.rma.ac.be \\ $\dagger$ CREA, Ecole de l'Air, Salon de Provence, France, jraout@cr-ea.net \\ $\ddagger$ ONERA, Salon de Provence, France, Serge.Roques@onera.fr \\ $\S$ Dept. of Electrical Engineering and Computer Science, University of Liège, Belgium, \\ Jacques.Verly@ulg.ac.be
}

\begin{abstract}
In this paper, we examine the feasibility of applying Space-Time Adaptive Processing (STAP) to bistatic passive radars using illuminators of opportunity. The transmitters considered are GSM base stations and are non-cooperative. Although STAP has been extensively applied to signals from pulse-Doppler radars, it was never applied to arbitrary signals arising from illuminators of opportunity. We show that by computing the appropriate mixing product, we essentially convert the signal of opportunity to a pulse-Doppler like signal, hence making the application of STAP to arbitrary signals straightforward. We finally confirm these theoretical results by using real measurements.
\end{abstract}

\section{INTRODUCTION}

Radars using illuminators of opportunity are inherently passive bistatic radars. The passivity of bistatic radars offers definitive advantages [1] among which low cost, low weight and enhanced radar cross-section for certain geometries. Moreover, stealth operations are possible since the receiver is totally passive.

Radars using illuminators of opportunity have already been studied. Signals provided by FM radio broadcast [2], satellites [3], [4], digital video broadcast (DVB-T) [5], and Global System for Mobile communications (GSM) base stations [6] have been considered. Arguments for the selection of the transmitter type include spatial and time coverage, power, central-frequency and bandwidth of the emitted signal, and shape of the ambiguity function. The bandwidth dictates the achievable range-resolution and the shape of the ambiguity function is decisive in determining the detection performance of the radar. In particular, signals from digital modulation (GSM, DVB) have much less range and Doppler ambiguities than other modulations [7], which makes them more suitable for passive radar. In this paper, we will consider GSM base stations as illuminators of opportunity. They have an ubiquitous spatial coverage, are permanent in time and have a thumbtack like ambiguity function due to the noise-like behavior of the GMSK modulation used. The main drawback of GSM base station signals is the small bandwidth [8] that yields a range resolution of about $1.8 \mathrm{~km}$. Thus a GSM-based radar will only be usable to perform moving target detection. The Doppler frequency resolution will only depend on the coherent integration time (CIT). A CIT of a few tenths of a second is easily achievable and yields a Doppler frequency resolution of about a few $\mathrm{Hz}$.
Space-Time Adaptive Processing (STAP) is typically used to filter out (clutter-)interferences in GMTI radars in order to detect slow-moving targets. STAP offers a benefit over separate spatial and temporal processing when there is a coupling between the clutter signal direction of arrival (DOA) and its Doppler frequency. STAP consists in performing a joint spatio-temporal optimum filtering of the signal in order to reject interference (clutter) contributions [9], [10].

In the STAP literature, it is assumed that the available signal is formed by the echoes from a pulse-Doppler radar. This paper shows how STAP can be applied to other types of signals and in particular to the noise-like GSM signals. The acquisition of a GSM signal and the signal itself are described in Section II. Section III shows how noise-like signals must be handled in order to make STAP processing feasible. Section IV details the issues involved in the estimation of the covariance matrix required to perform STAP processing and Section V shows end-to-end results for real signals.

\section{SIGNAL ACQUISITION AND PRE-PROCESSING}

A block diagram of a passive GSM-based radar receiver is depicted in Fig. 1. We use a two-channel receiver, the two antennas being arranged to form an array. The array is oriented such that the broadside direction is pointing to the targets. After amplification by the low noise amplifier

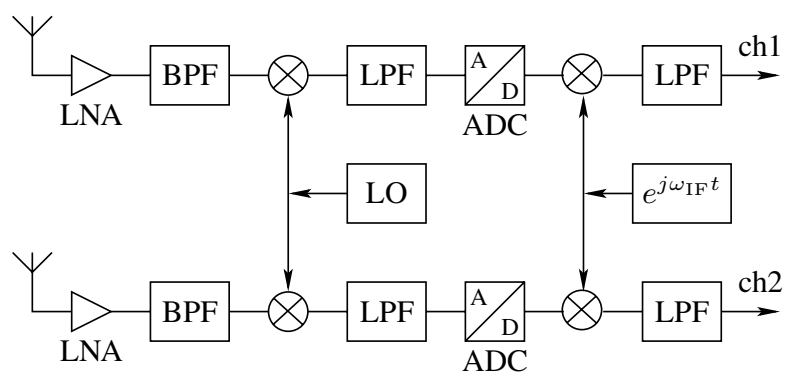

Fig. 1. Block diagram of the receiver.

(LNA) and filtering by the band pass filter (BPF) to keep only the GSM downlink band, the signal is down-converted to intermediate frequency and sampled. Once sampled, the received signal is further down-converted using digital downconversion (DDC). Performing the last down-conversion step 
numerically eliminates imbalances between the in-phase and the in-quadrature channels.

To correct for possible asymmetry between the two channels, a calibration step is required. The calibration is also used to measure the phase center of the antennas in order to extract correct direction information from the measurements.

Since the bandwidth of the receiver is much larger than that of one GSM channel, the signals from several GSM base stations can be received at once. A typical spectrum of the acquired signal is depicted in Fig. 2. The different GSM

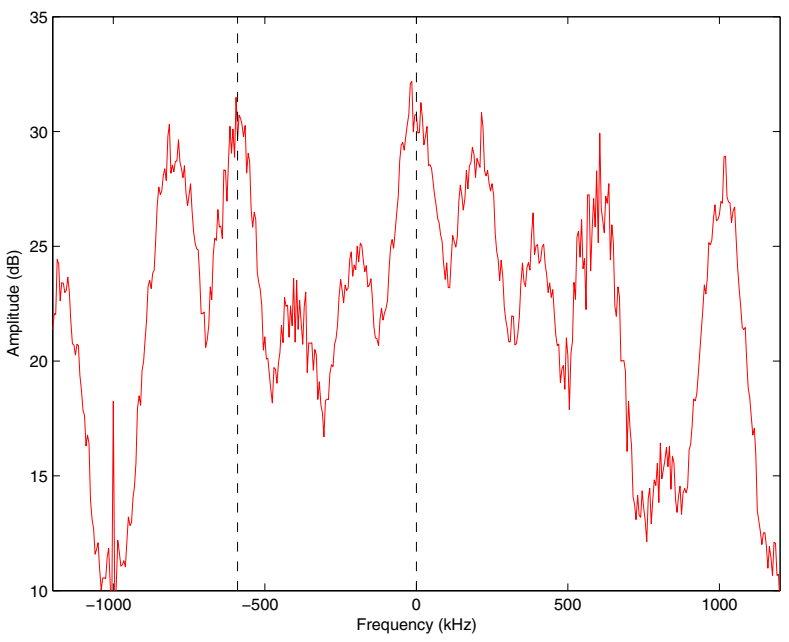

Fig. 2. Spectrum of the received signal

downlink channels are clearly visible. The two most powerful channels, located around $0 \mathrm{~Hz}$ and $-600 \mathrm{kHz}$, correspond to two different base stations.

The received signal contains the direct signal coming from the GSM base-station transmitter and the echoes of the GSM base-station signal backscattered by the vegetation, buildings and targets. To be able to perform the coherent processing, it is fundamental to know the reference signal broadcast by the GSM base-station. [11] describes a method able to blindly extract the reference signal from an array of sensors. The method uses adaptive beamforming. Indeed, to avoid artifacts, it is essential that the reference signal does not contain any echoes from any target. If the reference signal would contain echoes from targets, the concerned targets would be attenuated by the echo cancellation processing. In the remaining of this paper, we will assume that the reference signal, denoted $x_{\text {ref }}$, is available.

\section{GENERALIZATION OF STAP TO NOISE-LIKE SIGNALS}

The detection of targets in echo signals is typically performed by using a matched filter. The matched filtering can be generalized in the form of the range-Doppler diagram [12], [13]

$$
\chi\left(\nu_{d}, n\right)=\sum_{k} x(k) x_{\mathrm{ref}}^{*}(k-n) e^{-j 2 \pi \nu_{d} k}
$$

where $x(k)$ are the samples of the signal containing echoes from the potential target, the clutter, and the direct path signal; $n$ denotes the range at which the correlation is computed; and $\nu_{d}$ is the reduced Doppler frequency. As noted in [13], [14], this can be seen as the Fourier transform of the mixing product

$$
x_{m}(k)=x(k) x_{\mathrm{ref}}^{*}(k-n) .
$$

This mixing can be seen as a generalization of the usual heterodyne down-mixing. In this way, the random phase variation of $x(k)$ along $k$ due to the emitted signal $x_{\text {ref }}(k-n)$ is removed. Further, this formulation is useful to implement adaptive filtering instead of the classical Fourier-based filter banks [11].

To perform STAP, measurements obtained from $N$ different channels are required. Let us denote by $\mathbf{x}(k)$ the vector containing the $N$ signal samples at time $k T_{s}$ where $T_{s}=1 / f_{s}$ is the temporal sampling interval and $f_{s}$ is the temporal sampling frequency. Let the mixing product resulting from mixing the signal from each channel with a time-delayed version of the reference signal $x_{\text {ref }}(k-n)$ be denoted by

$$
\mathbf{x}_{m}(k ; n)=\mathbf{x}(k) \circ\left(\mathbf{1} \otimes x_{\mathrm{ref}}(k-n)\right)^{*}
$$

where 1 is a $N \times 1$ column vector with unit elements, $\otimes$ denotes the Kronecker product and $\circ$ the Hadamar (elementwise) product. Since the targets of interest induce a Doppler frequency that is much smaller than the sampling frequency $f_{s}$, the signal $\mathbf{x}_{m}(k)$ can be low-pass filtered and subsampled as suggested in [14]. Note that this subsampling does not affect the range-resolution of the radar.

Let us denote by

$$
\begin{aligned}
& \mathbf{x}_{\mathbf{s}}(n)=\left[\mathbf{x}_{m}^{T}(0 ; n), \mathbf{x}_{m}^{T}(S ; n),\right. \mathbf{x}_{m}^{T}(2 S ; n), \ldots, \\
&\left.\mathbf{x}_{m}^{T}((M-1) S ; n)\right]^{T}
\end{aligned}
$$

the lexically ordered subsampled signal, where $S$ is the subsampling factor. The number of temporal samples $M$ depends on the CIT and on the subsampling factor $S$. A typical value is $M=256$. The temporal Fourier transform processing that was applied to single-channel signals in eq. (1) can also be generalized to the spatial domain and applied to $\mathbf{x}_{\mathbf{s}}$ yielding

$$
y_{m}\left(\nu_{s}, \nu_{d} ; n\right)=\mathbf{v}^{\dagger}\left(\nu_{s}, \nu_{d}\right) \mathbf{x}_{\mathbf{s}}(n)
$$

where $\mathbf{v}$ is the spatio-temporal steering vector

$$
\mathbf{v}\left(\nu_{s}, \nu_{d}\right)=\mathbf{a}\left(\nu_{s}\right) \otimes \mathbf{b}\left(\nu_{d}\right) \text {. }
$$

In the case of an uniform linear array (ULA), the spatial steering vector is

$$
\mathbf{a}\left(\nu_{s}\right)=\left[1, e^{j 2 \pi \nu_{s}}, \ldots, e^{j 2 \pi \nu_{s}(N-1)}\right]^{T}
$$

where $\nu_{s}=\frac{d}{\lambda} \sin \theta$ is the reduced spatial frequency with $d$ the inter-element spacing, $\lambda$ the carrier wavelength and $\theta$ the incidence angle. The temporal steering vector is given by

$$
\mathbf{b}\left(\nu_{d}\right)=\left[1, e^{j 2 \pi \nu_{d}}, \ldots, e^{j 2 \pi \nu_{d}(M-1)}\right]^{T} .
$$

where $\nu_{d}=\frac{f_{D} S}{f_{s}}$ is the reduced Doppler frequency with $f_{D}$ the Doppler frequency of the target and $f_{s}$ the sampling frequency of the acquisition. The size of the spatio-temporal steering vector depends on the number of channels $N$ and on the 
number of temporal samples $M$ considered in the subsampled signal.

This is illustrated on Fig. 3, where the matched filter

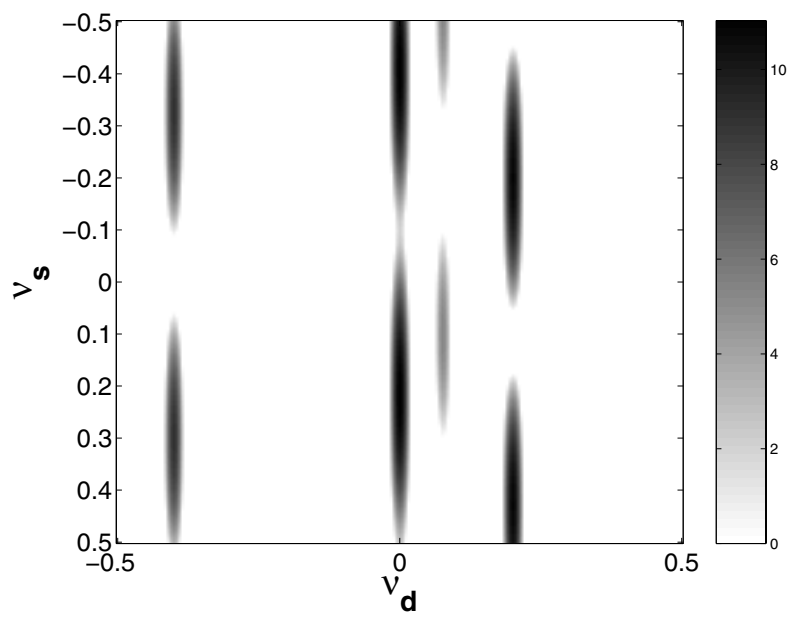

Fig. 3. Matched filter output on a signal containing simulated targets.

output $y_{m}$ of a simulated signal is shown. The signal was generated by adding simulated targets to real clutter measurements. The spatial (direction) ambiguities results from the use of a 2-elements antenna array with a distance of about $0.8 \lambda$ between the two elements. The targets are simulated by adding time-delayed and frequency-delayed versions of the reference signal to the signals from the spatial channels. Targets all have the same amplitude except the last one with half this amplitude and are located at $\left(\nu_{s}, \nu_{d}\right)=$ $\{(0.3,-0.4),(-0.2,0.2),(0.1,0.08)\}$. Note that in addition to the targets, a relatively strong direct path signal is present at $\left(\nu_{s}, \nu_{d}\right)=(-0.2,0)$.

Similarly, we can apply an adaptive filter

$$
y\left(\nu_{s}, \nu_{d} ; n\right)=\mathbf{w}^{\dagger}\left(\nu_{s}, \nu_{d}\right) \mathbf{x}_{\mathbf{s}}(n)
$$

where $y$ is the output of the filter at these frequencies, and the filter $\mathbf{w}$ that rejects the interferences and the noise in an optimum way is given by [9], [15]

$$
\mathbf{w}\left(\nu_{s}, \nu_{d}\right)=R^{-1} \mathbf{v}\left(\nu_{s}, \nu_{d}\right)
$$

where $R$ is the covariance matrix of the interference-plus-noise data and $\mathbf{v}$ the spatio-temporal steering vector (6).

\section{ESTIMATION OF THE COVARIANCE MATRIX}

The interference covariance matrix $R$ required to compute the optimum filter (10) is defined as

$$
R=E\left[\mathbf{x}_{s_{i+n}} \mathbf{x}_{s_{i+n}}^{\dagger}\right]
$$

where $\mathbf{x}_{s_{i+n}}$ is the mixed, low-pass filtered, subsampled and lexically ordered signal, containing only interference and noise.

The expectation operator $E$ is typically replaced by a sum over data samples taken at different ranges [16], i.e. the sample covariance matrix (SCM). The estimation obtained will be unbiased only if the averaged data samples are independent and identically distributed. In bistatic configurations, the clutter power spectrum locus is known to exhibit a range-dependency. Hence, independently of possible clutter inhomogeneities, the conditions for unbiased estimation are typically not verified. However, in the configuration considered here, i.e. a static transmitter and a receiver located on the ground, it was shown in [17] that the clutter power spectrum locus is independent of the range. This means that in the considered configuration, no geometry-induced range dependence of the clutter statistics will be present.

To obtain a useful estimation, a relatively large number of samples needs to be averaged [16]. Taking into account an optimistic reach of about $20 \mathrm{~km}$ and a range-resolution of $2 \mathrm{~km}$, only 10 independent samples are available. Note that this is an overly optimistic figure since it does not take into account range inhomogeneities. To cope with the low number of samples available, diagonal loading (DL) is typically performed, leading to the so-called SCM+DL estimation method. The low-rank nature of $R$ can be exploited to further reduce the number of samples required to perform a useful estimation. In particular, a method based on the extraction of the principal components of $R$ was proposed [18]. The method was subsequently enhanced [18] by taking into account the modeling of decorrelation effects due, for instance, to internal clutter motion (ICM) using a covariance matrix taper (CMT). This yields the PC+CMT+DL method. Although many other estimation methods exist, a complete discussion of covariance matrix estimation methods applicable in the current scenario is outside the scope of this paper and we will limit ourself to the methods described above. Figure 4 presents the performances of the estimation methods discussed above in terms of SINR loss [19]. These results were obtained by using real data (the actual scenario considered is detailed in section V). The reference for comparison is a theoretical covariance matrix model obtained by assuming a zero-Doppler clutter with a small amount of ICM. As can be seen, the SINR loss corresponding

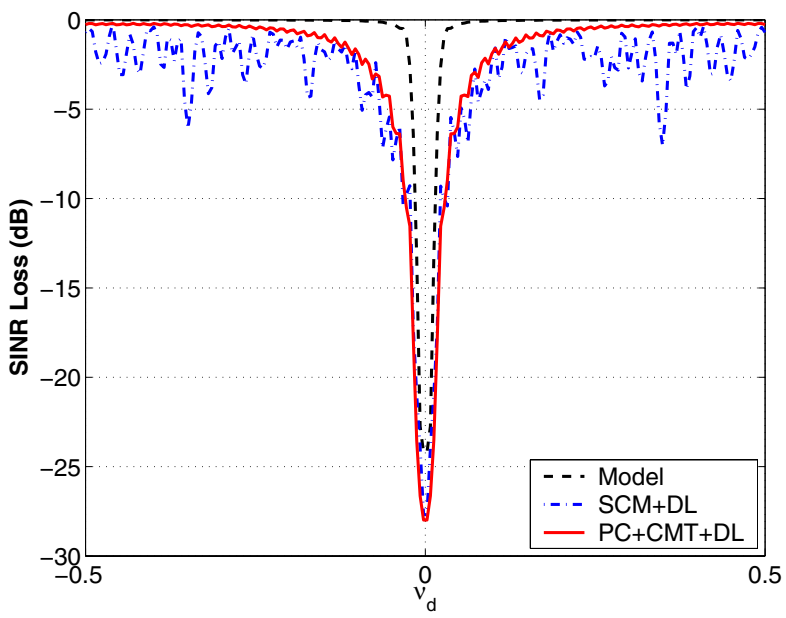

Fig. 4. Comparison of the performance of various covariance matrix estimation methods (cut at zero spatial frequency $\nu_{s}=0$ ).

to the $\mathrm{SCM}+\mathrm{DL}$ covariance matrix estimation is relatively high 
even at non-zero Doppler frequencies. This is due partly to the low number of data samples available and partly to the Doppler sidelobes of the ambiguity function inherent to the noise-like signal used. Although the $\mathrm{PC}+\mathrm{CMT}+\mathrm{DL}$ estimation method performs better, the increase in minimum detectable velocity is only marginally better. It should further be noted that while it is desirable to remove estimation artifacts due to a low number of data samples, the effect of Doppler sidelobes should be kept and will impact detection performance. Other methods, such as CLEAN-based methods [11], [20] suffer less from the sidelobes of the ambiguity function.

\section{END-TO-END PERFORMANCE}

End-to-end performance results are obtained by computing $y\left(\nu_{s}, \nu_{d} ; n\right)$ for a particular range $n$ and for all possible spatial and temporal frequencies $\nu_{s}$ and $\nu_{d}$.

\section{A. Simulated data}

In this section we present results based on simulated data. The scenario considered is depicted in Fig. 5 and involves a static GSM base station and a receiver moving at a speed of $10 \mathrm{~m} / \mathrm{s}$. The receiving array is an $\lambda / 2$-spaced 8 -elements ULA and is oriented to be forward-looking. The transmit and the

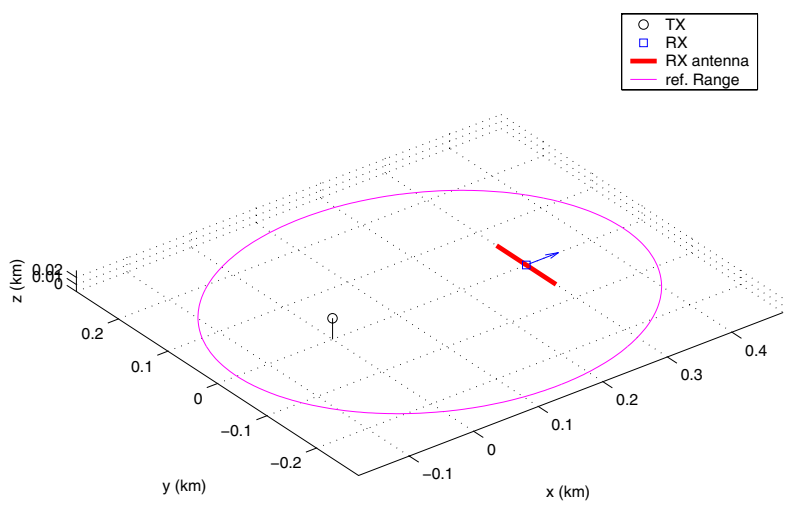

Fig. 5. Scenario considered with the isorange considered drawn as a solid line.

receive antennas have an omnidirectional radiation pattern to exacerbate the influence of the clutter. In practice, the radiation pattern of GSM base-stations is far from omnidirectional.

Bistatic scenarios typically involve a geometry-induced range-dependence of the clutter statistics. However, this particular case where the transmitter is static and the receiver is located on the ground does not exhibit any geometry-induced range-dependence as shown in [17]. This is illustrated in Fig. 6 where the clutter power spectrum locus is depicted for different ranges.

Figure 7 depicts the power spectrum of the mixed signal $\mathbf{x}_{m}$ at the range of interest. The clutter power spectrum locus is plot as a thin line. The contribution of the clutter along the clutter power spectrum locus is clearly visible.

We considered a modeled interference-plus-noise covariance matrix for the computation of the adapted matched filter. The power spectrum of the considered covariance matrix

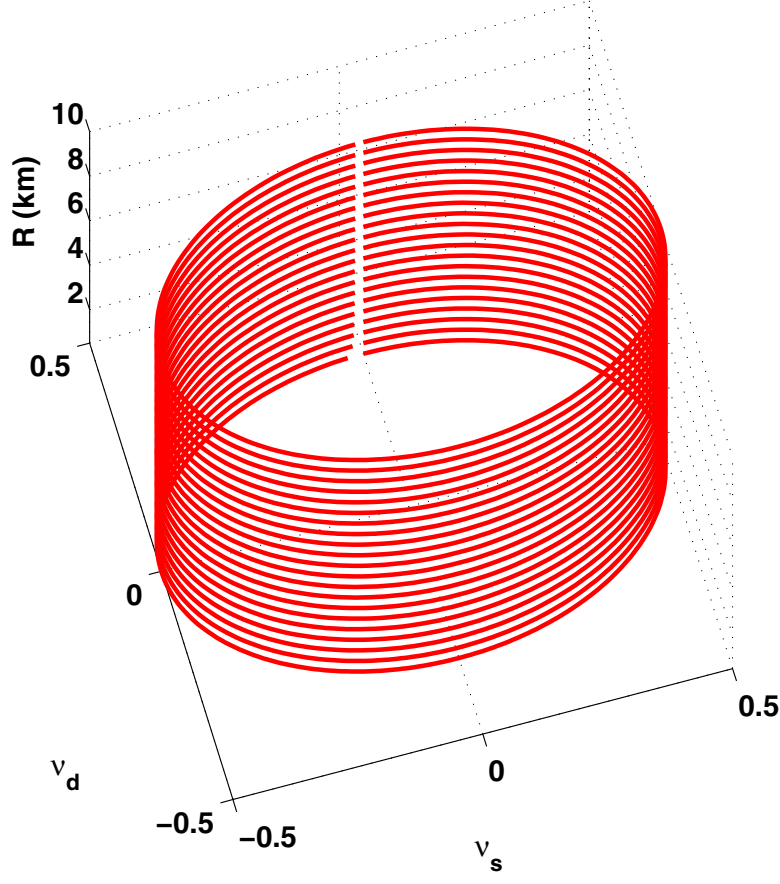

Fig. 6. Clutter power spectrum locus in function of the range.

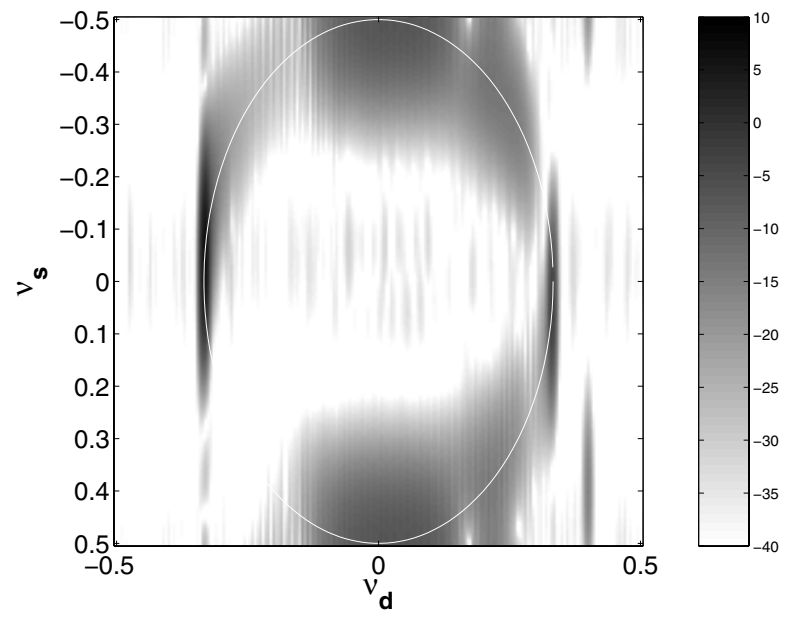

Fig. 7. Matched filter output and clutter power spectrum locus.

is illustrated in Fig. 8. Again, the power spectrum of the covariance matrix is located along the clutter power spectrum locus.

The result $y$ of the application of the optimum filter to the mixed signal $\mathbf{x}_{m}$ is illustrated in Fig. 9, the thin solid line being the clutter power spectrum locus. As can be seen, the clutter contribution is filtered out, leaving the target standing out at $\left(\nu_{s}, \nu_{d}\right)=(0.4,0.4)$.

\section{B. Measured data}

The results presented here correspond to real measurements. The geometric configuration of the transmitter, receiver and target is illustrated in Fig. 10. The receiving antenna array is static and has 2 elements separated by about $0.8 \lambda$. The 


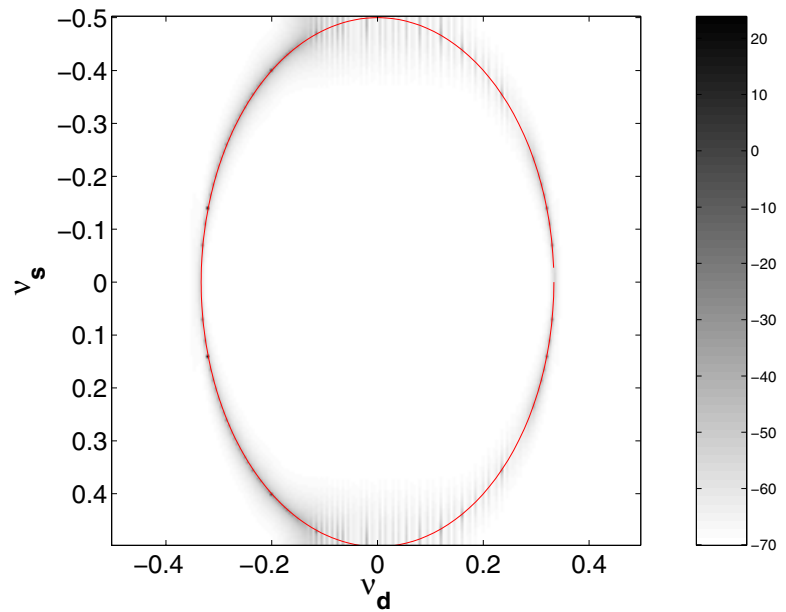

Fig. 8. Covariance matrix power spectrum.

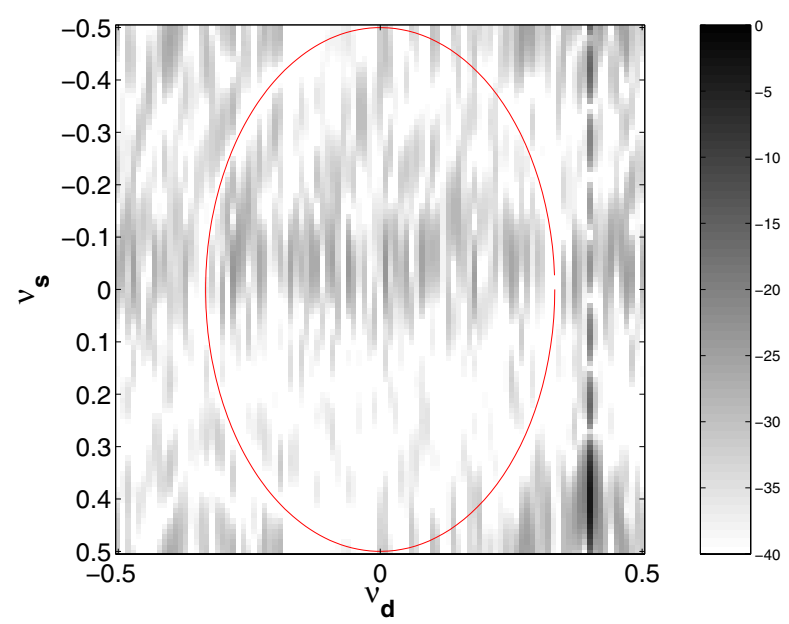

Fig. 9. Adapted matched filter output and clutter power spectrum locus.

scenario involves a cooperative vehicle (a small van) approaching the receiver and yielding a Doppler frequency of about $-40 \mathrm{~Hz}$. With this Doppler frequency and taking into account the frequency resolution, the vehicle signature is buried in the sidelobes of the (untapered) matched filter $\mathbf{w}=\mathbf{v}$. A tapered matched filter yields the classical angle-Doppler diagram of Fig. 11. By using the proposed STAP approach, the contributions due to clutter (including both the static part and the small ICM components) can be removed, leaving the target echo standing out as can be seen at $\left(\nu_{s}, \nu_{d}\right)=(0.4,-0.07)$ in Fig. 12; the other signatures are due either to reflexions or to other (uncooperative) targets. The adapted filter is obtained from (10) using a modeled covariance matrix involving ICM.

Figure 13 presents a cut along $\nu_{s}=0.4$ in the angle-Doppler diagrams presented in Fig. 11 and Fig. 12. As can be seen, the effect of the adapted filter is essentially to remove the components due to clutter, located around zero-Doppler. The amplitude of the signature of the vehicle at $\nu_{d}=-0.07$ is smaller for the tapered matched filter than for the (untapered) adapted filter. This is due to the tapering losses.

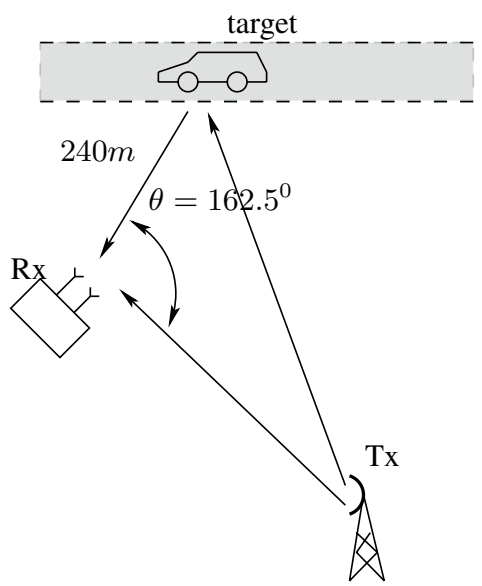

Fig. 10. Geometric configuration for the real measurement.

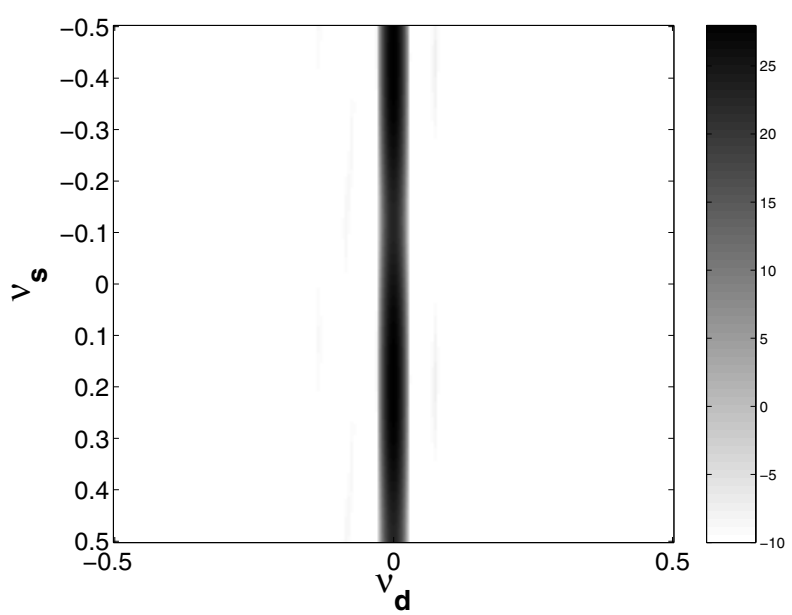

Fig. 11. Angle-Doppler diagram obtained by using a classical matched filter.

It should be noted that, since the transmitter and the receiver are both static, the clutter angle-Doppler diagram does not exhibit the classical coupling. Hence space-time processing is,

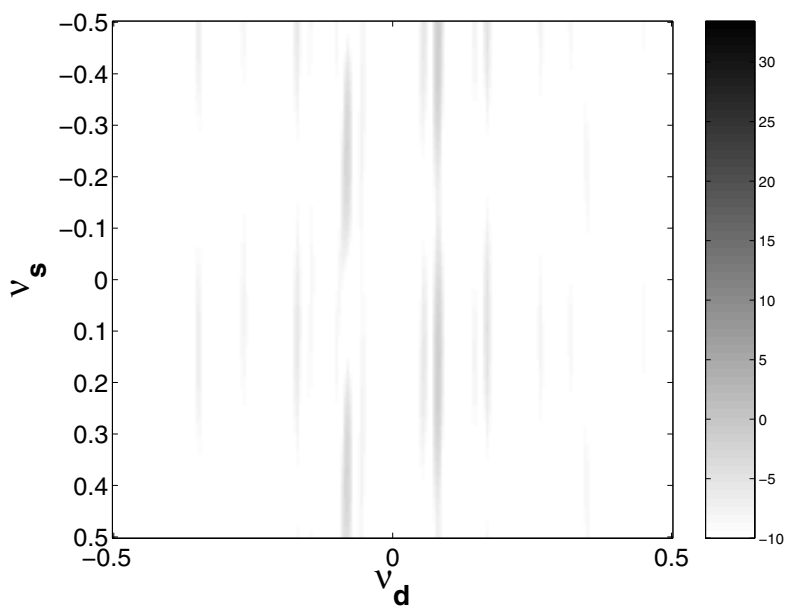

Fig. 12. Angle-Doppler diagram obtained by using an adapted matched filter. 


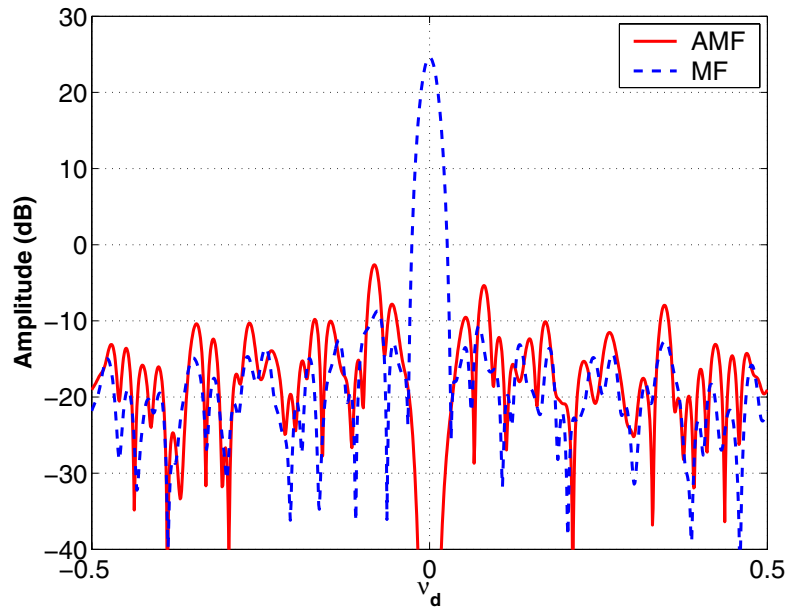

Fig. 13. Cut along $\nu_{s}=0.4$ in the angle-Doppler diagrams of Fig. 11 and Fig. 12 comparing the behavior of the classical matched filter (MF) and the proposed adapted matched filter (AMF).

in this particular scenario, not really required and a temporal processing would be sufficient. This can nevertheless be seen as a degenerate case of joint space-time processing.

\section{CONCLUSION}

In this paper, we propose a generalization of space-time adaptive processing to noise-like signals. We show that the estimation of the covariance matrix of the interference-plusnoise samples is challenging due to the presence of range and Doppler ambiguities. Finally, we show the applicability of this generalization to a passive radar using GSM base stations as illuminators of opportunity. The results obtained on real data show that the proposed method effectively filters out the clutter signal.

The computation of the optimum filter requires an estimate of the interference plus noise covariance matrix. Obtaining an accurate estimate of this matrix from noise-like signals is, however, still challenging.

\section{REFERENCES}

[1] W. L. Melvin, "Issues in bistatic STAP," in Proceedings of the 32nd Southeastern Symposium on System Theory, (Tallahassee, FL), Mar. 2000.
[2] H. Zheng, F. Li, J. Luo, and J. Lu, "Bistatic radar experiment based on FM broadcast transmitter," in Proc. RADAR'04, (Toulouse, France), Oct. 2004.

[3] J. Horner, K. Kubik, B. Mojarrabi, I. D. Longstaff, E. Donskoi, and M. Cherniakov, "Passive bistatic radar sensing with LEOS based transmitters," in Proceedings of IGARSS 02, pp. 438-440, June 2002.

[4] M. Cherniakov, T. Zeng, and E. Plakidis, "Galileo signal-based bistatic system for avalanche prediction," in Proceedings of IGARSS 03, pp. 784786, July 2003

[5] R. Saini, M. Cherniakov, and V. Lenive, "Direct path interference suppression in bistatic system: DTV based radar," in Proc. of the International Radar Conference 2003, pp. 309-314, Sept. 2003.

[6] D. K. P. Tan, H. Sun, Y. Lui, M. Lesturgie, and H. Chan, "Passive radar using global system for mobile communication signal: theory, implementation and measurements," IEE Proceedings - Radar, Sonar and Navigation, vol. 152, pp. 116-123, June 2005.

[7] H. D. Griffiths and C. J. Baker, "Measurement and analysis of ambiguity functions of passive radar transmissions," in Proc. of the International IEEE Radar Conference 2005, pp. 321-325, May 2005.

[8] D. K. P. Tan, H. Sun, Y. Lu, and W. Liu, "Feasibility analysis of GSM signal for passive radar," in Proc. of the International IEEE Radar Conference 2003, pp. 425-430, May 2003.

[9] R. Klemm, Principles of space-time adaptive processing. UK: The Institution of Electrical Engineers (IEE), 2002.

[10] J. R. Guerci, Space-Time Adaptive Processing for Radar. Norwood, MA: Artech House, 2003.

[11] M. Kubica, V. Kubica, X. Neyt, J. Raout, S. Roques, and M. Acheroy, "Optimum target detection using emitters of opportunity," in Proceedings of the IEEE Radar Conference, (Verona, NY), Apr. 2006.

[12] K. Kulpa, "Continuous wave radars - monostatic, multistatic and network," in Advances in Sensing with security applications, (Il Ciocco, Italy), NATO Advanced Study Institute, July 2005.

[13] S. R. J. Axelsson, "On the theory of noise Doppler radar," in Proc. of IGARSS 2000, pp. 856-860, July 2000.

[14] S. Stein, "Algorithms for ambiguity function processing," IEEE Transactions on Acoustics, Speech and Signal Processing, vol. 29, pp. 588-599, June 1981.

[15] J. Ward, "Space-time adaptive processing for airborne radar," Tech. Rep. 1015, MIT Lincoln Laboratory, Lexington, MA, Dec. 1994.

[16] I. S. Reed, J. D. Mallett, and L. E. Brennan, "Rapid convergence rate in adaptive arrays," IEEE Transactions on Aerospace and Electronic Systems, vol. 10, pp. 853-863, Nov. 1974.

[17] X. Neyt, P. Ries, J. G. Verly, and F. D. Lapierre, "Registration-based range-dependence compensation method for conformal-array STAP," in Proc. Adaptive Sensor Array Processing Workshop, (MIT Lincoln Laboratory, Lexington, MA), June 2005.

[18] J. R. Guerci and J. S. Bergin, "Principal components, covariance matrix tapers, and the subspace leakage problem," IEEE Transactions on Aerospace and Electronic Systems, vol. 38, pp. 152-162, Jan. 2002.

[19] D. G. Manolakis, V. K. Ingle, and S. M. Kogon, Statistical and Adaptive Signal Processing. USA: McGraw Hill, 1998.

[20] G. R. Legters, "Using a plane-wave signal model to suppress airborne GMTI radar clutter and calibrate the array," in Proceedings of KASSPER 03, (Las Vegas, NV), pp. 283-288, Apr. 2003. 